\title{
Réfugiés et déplacés dans l'Est du Tchad
}

De l'intervention humanitaire à la sécurisation militaire

\section{Johanne Favre}

\section{OpenEdition}

1 Journals

Édition électronique

URL : http://journals.openedition.org/echogeo/2061

DOI : 10.4000/echogeo.2061

ISSN : 1963-1197

\section{Éditeur}

Pôle de recherche pour l'organisation et la diffusion de l'information géographique (CNRS UMR 8586)

\section{Référence électronique}

Johanne Favre, «Réfugiés et déplacés dans l'Est du Tchad », EchoGéo [En ligne], Sur le Vif, mis en ligne le 13 novembre 2007, consulté le 01 mai 2019. URL : http://journals.openedition.org/echogeo/2061 ; DOI : 10.4000/echogeo.2061

Ce document a été généré automatiquement le 1 mai 2019.

\section{(c) (i) ()}

EchoGéo est mis à disposition selon les termes de la licence Creative Commons Attribution - Pas d'Utilisation Commerciale - Pas de Modification 4.0 International 


\section{Réfugiés et déplacés dans l'Est du Tchad}

De l'intervention humanitaire à la sécurisation militaire

Johanne Favre

1 Le 16 octobre 2007, alors que l'Union Européenne vient d'autoriser l'envoi au Tchad et en RCA d'une force d'environ 4000 soldats, le gouvernement tchadien rétablit l'état d'urgence dans le nord et l'est du pays, suite à une recrudescence d'affrontements qualifiés d'» interethniques ».

2 Au vrai, la situation dans l'est tchadien n'a cessé de se dégrader depuis le déclenchement de la rébellion du Darfour, de l'autre côté de la frontière soudanaise, en février 2003. L'installation des réfugiés du Darfour dans 12 camps au Ouaddaï, dans le Wadi Fira et dans l'Ennedi, a fait naître des tensions avec les populations locales, dans un contexte de rareté des ressources et d'antagonismes ethniques. A partir d'octobre 2005, la région redevient le théâtre de rébellions hostiles au président tchadien Idriss Déby. Un an plus tard, les débordements de la guerre du Darfour, conjugués aux effets d'une crise politique interne au Tchad, provoquent l'embrasement de la région. 180000 Tchadiens sont chassés de chez eux par la violence. 


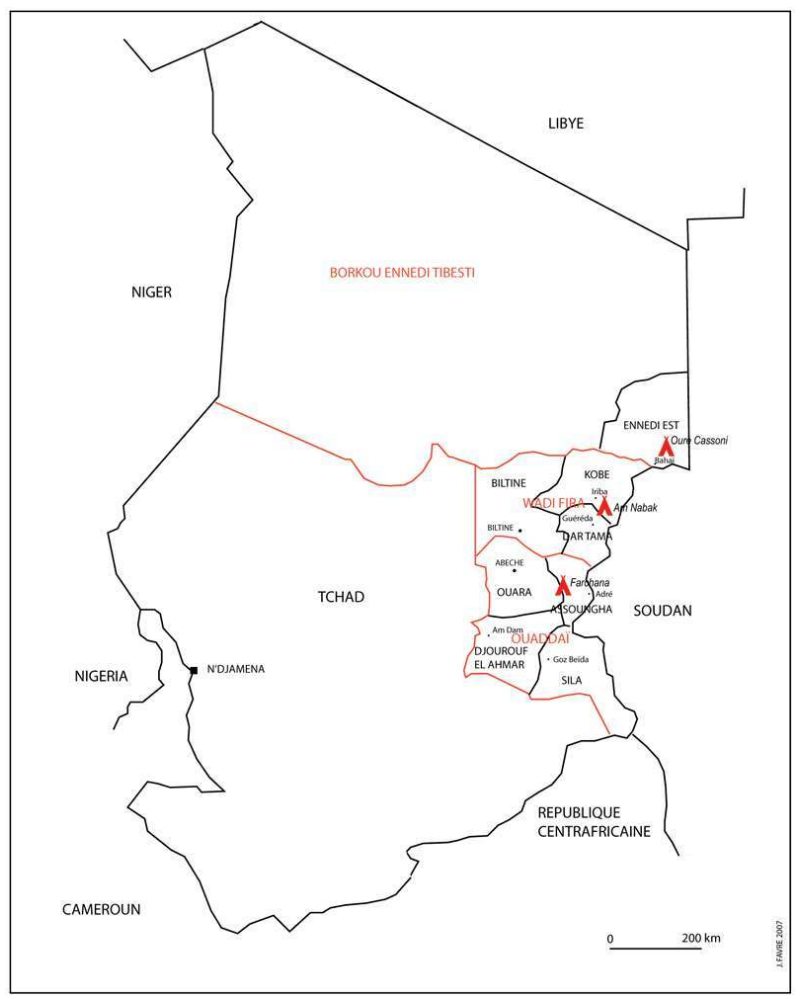

3 La résolution 1778 adoptée par le Conseil de sécurité de l'ONU le 25 septembre 2007 approuve l'envoi dans l'est tchadien d'une "présence multidimensionnelle » comprenant une mission des Nations Unies (la MINURCAT) et une force militaire européenne (l'EUFOR Tchad/RCA). Son objectif n'est pas le maintien de la paix - de quelle paix ? - mais la protection et le retour des réfugiés et des déplacés, et la sécurisation des interventions humanitaires. Une mission qui suscite espoirs et craintes.

1. Des camps de réfugiés dans un environnement contraignant

4 Les premiers sont arrivés en avril 2003. Les réfugiés soudanais sont aujourd'hui plus de $230000^{1}$ dans l'est du Tchad. Leur présence augmente d'un quart la population de la région. $85 \%$ d'entre eux sont regroupés dans 10 camps implantés en milieu sahélien ou sahélo-saharien. Or le désert, dans lequel les hommes et les troupeaux ne survivent que par la mobilité, est peu propice à l'implantation durable de ces véritables « villes ».

L'eau est la contrainte principale. Dans plusieurs camps, les réfugiés reçoivent une quantité d'eau journalière très inférieure à la norme de 15 litres. Le camp d'Am Nabak est approvisionné par camions-citernes à partir d'Iriba.

6 En saison des pluies, c'est l'excès d'eau qui entrave l'accès aux camps : le Programme Alimentaire Mondial (PAM), dont les convois pour approvisionner cette région enclavée partent de Douala ou de Benghazi, doit « prépositionner » des vivres pour plusieurs mois à proximité des camps quand les pistes sont rendues impraticables par les crues des ouadis (les cours d'eau temporaires). En août 2007, des pluies exceptionnelles ont détruit des habitations dans les villages et les camps au sud du Ouaddaï; des inondations ont aussi affecté le camp le plus septentrional, celui d'Ouré Cassoni, dans l'Ennedi Est.

7 Trouver du bois est l'autre source majeure de préoccupation. Elle cristallise les conflits entre réfugiés et populations locales. Autour de chaque camp s'étend une auréole de 
déforestation. Les solutions mises en œuvre par les organisations internationales (collecte et distribution de bois mort, essais ponctuels de foyers améliorés et de cuiseurs solaires, plantations d'arbres) ne suffisent pas à empêcher la dégradation.

8 La présence des troupeaux des réfugiés donne aussi lieu à des heurts avec les autochtones, d'autant plus que des terres sont attribuées aux Soudanais qui souhaitent cultiver. Les vallées des ouadis, à la fois terres agricoles, pâturages et source d'eau, font l'objet d'une âpre concurrence.

En mars 2004, une étude environnementale menée par le HCR préconisait de déplacer les camps de 200 à 300 kilomètres vers le sud. Mais il a fallu prendre en compte des contentieux interethniques anciens, et finalement les réfugiés, qui appartiennent pour la plupart à des communautés présentes des deux côtés de la frontière, ont été transférés dans des camps situés à la même latitude que leur zone de provenance.

L'afflux de l'aide internationale dans cette région pauvre déstabilise l'économie et la société. Les populations locales, dont certaines sont venues en aide aux réfugiés avant l'arrivée des organisations internationales, sont plus démunies que leurs hôtes désormais nourris, soignés et éduqués dans les camps. Les projets qui leur sont peu à peu destinés sont inaptes à répondre à l'ensemble des besoins.

2. Le retour des rébellions tchadiennes

11 Le Tchad et le Soudan se livrent une guerre par rébellions interposées. Le président tchadien Idriss Déby, un Zaghawa, est redevable à son homologue soudanais Omar ElBéchir de son accession au pouvoir en 1990. Mais les Zaghawa tchadiens soutiennent dès le début leurs frères soudanais entrés en rébellion contre le régime de Khartoum. Les camps de l'est tchadien constituent pour ces derniers un «sanctuaire humanitaire» dans lequel ils trouvent le repos et les vivres (Jean et Rufin, 1996).

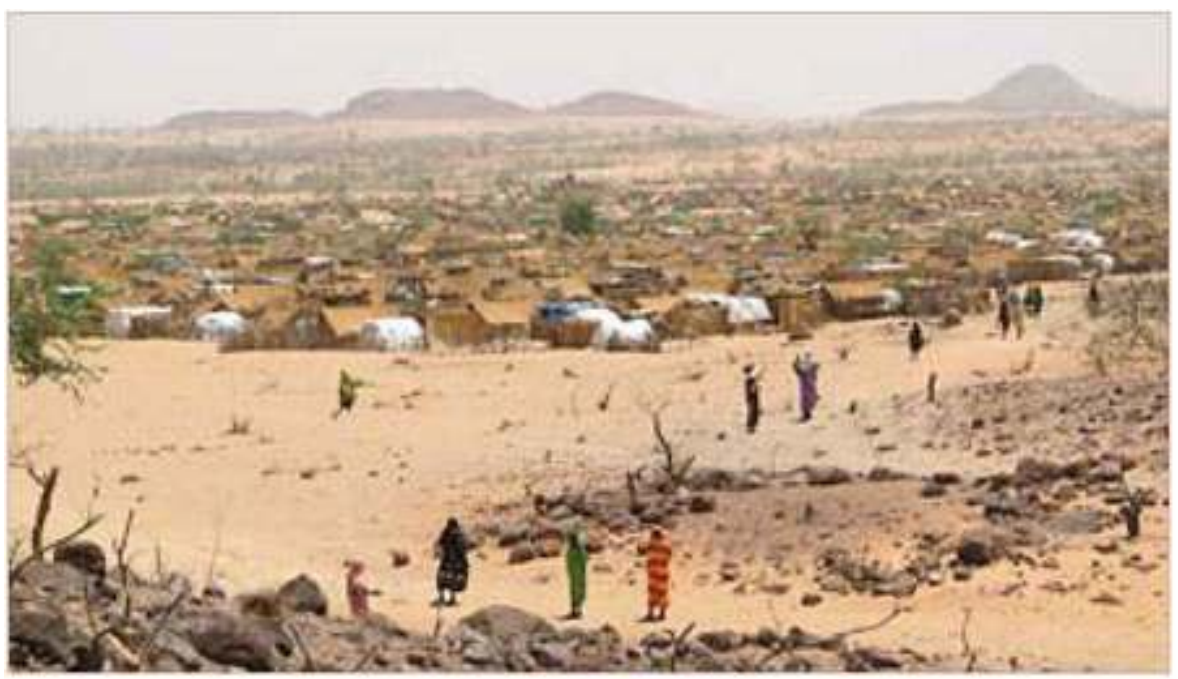

1. Le camp de Farchana, à 110 km à l'est d'Abéché. Premier camp ouvert par le HCR le 17 janvier 2004, il héberge aujourd'hui près de 20000 réfugiés soudanais 


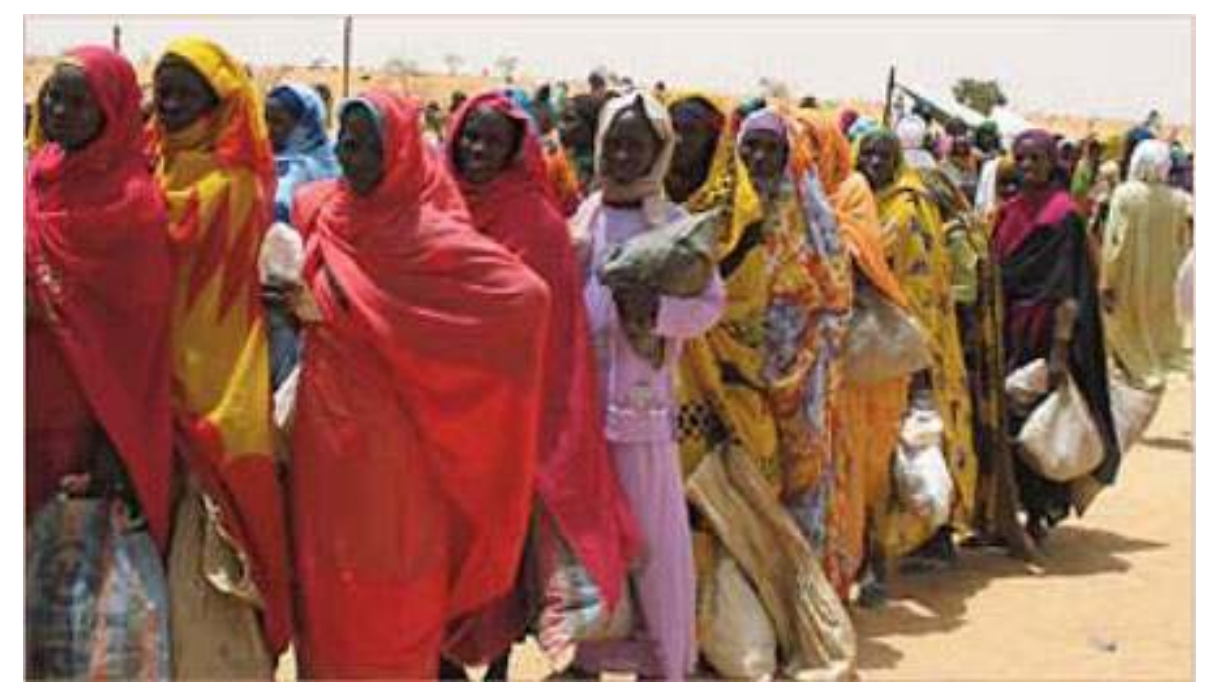

2. Distribution alimentaire dans le camp de Touloum.

L'aide apportée aux réfugiés soudanais par les organisations internationales crée des tensions avec des populations locales démunies.

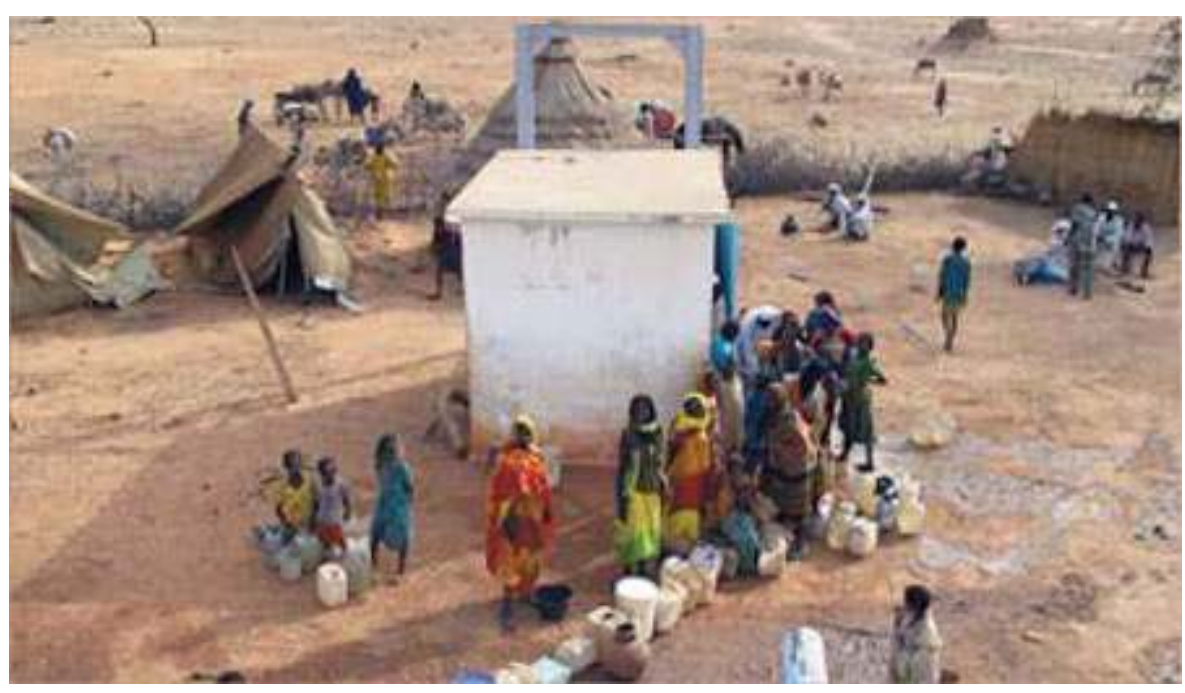

3. Un forage près de Goz Beïda.

Le réseau d'eau de la ville de Goz Beida est utilisé pour alimenter le camp de Djabal. Le forage est un lieu de disputes entre les réfugiés et les populations avoisinant le camp

12 En guise de riposte, le Soudan réactive son soutien aux rebelles tchadiens. Et l'on assiste à une prolifération de mouvements rebelles aux acronymes changeants, aux fusions éphémères. Le régime négocie des ralliements individuels.

Mahamat Nour, un Tama originaire de Guéréda, est l'un de ceux qui passent plusieurs fois de la rébellion aux responsabilités officielles. En octobre 2005, il fonde avec l'appui de Khartoum son propre mouvement, le RDL (Rassemblement pour la démocratie et la liberté), qui attaque la ville frontalière d'Adré le 18 décembre. Il est à la tête du FUC (Front uni pour le changement) quand il conduit en avril 2006 une offensive éclair de 800 kilomètres sur N'Djamena. Dans la capitale, la bataille fait 400 morts. Le FUC se divise. Sous l'égide de la Libye, Mahamat Nour se rallie au régime en décembre 2006. En mars 2007, il devient ministre de la Défense nationale.

Mais on ne peut interpréter la recrudescence des rébellions tchadiennes comme la seule conséquence du conflit du Darfour. La crise tchadienne résulte de l'accaparement du 
pouvoir et des ressources par le cercle dirigeant. A l'intérieur même de ce cercle, la prédation exercée par certains a accru les inégalités (Marchal, 2006). Idriss Déby a bloqué le jeu politique en modifiant la Constitution pour briguer un troisième mandat. Réélu en mai 2006, il utilise la manne pétrolière, non pour le développement du pays - comme le prévoyait la loi négociée avec la Banque mondiale sur la gestion des revenus pétroliers - mais pour des achats d'armes.

Ses adversaires les plus sérieux sont des proches: les jumeaux Erdimi, ses neveux, magnats du coton et du pétrole; Mahamat Nouri, ex-ministre et ex-ambassadeur du Tchad. Les mouvements rebelles créés par ceux-ci (le RFC, Rassemblement des forces pour le changement, et l'UFDD, Union des forces pour la démocratie et le développement) font partie des quatre qui ont signé le 25 octobre 2007 à Syrte un accord avec le gouvernement tchadien. Mais les motifs de griefs demeurent sur le terrain.

3. Les populations civiles dans la tourmente

$16 \mathrm{Au}$ Dar Tama, règne un antagonisme ancien entre Tama et Zaghawa: les premiers accusent les seconds d'avoir profité de leur position dominante pour les dépouiller de leur bétail. La présence de Tama dans la rébellion tchadienne dégrade les relations entre les deux communautés. A partir d'août 2006, des attaques de civils tama par des Zaghawa vident plusieurs villages autour de Guéréda. Le ralliement de Mahamat Nour donne lieu à des formes de vengeance qui perpétuent la violence.

17 Mais c'est dans la région du Ouaddaï que se manifeste, à partir d'octobre 2006, la " darfourisation $»^{2}$ de l'est tchadien. Des violences interethniques à grande échelle opposent désormais des «tribus arabes» et «non arabes ». Des milices d'autodéfense regroupant des membres d'ethnies « africaines» (Massalit, Dadjo...) se sont formées pour lutter contre les attaques des groupes "arabes", lesquels comprennent en fait des Soudanais et des Tchadiens, arabes mais aussi « ouaddaïens » (Tama, Mimi, Maba).

Le gouvernement tchadien a encouragé la formation de ces milices d'autodéfense, qui se font appeler Toro Boro, comme les rebelles soudanais qui les soutiennent. Dans le sud du Ouaddaï, l'entente entre les communautés est brisée. Des dizaines de villages sont brûlés, des atrocités sont commises contre des nouveaux-nés ou des 
infirmes. On dénombre jusqu'à 5000 victimes. Cinq des neufs cantons du département du Sila sont vidés de leur population. Des milliers de Tchadiens s'enfuient au Darfour voisin³

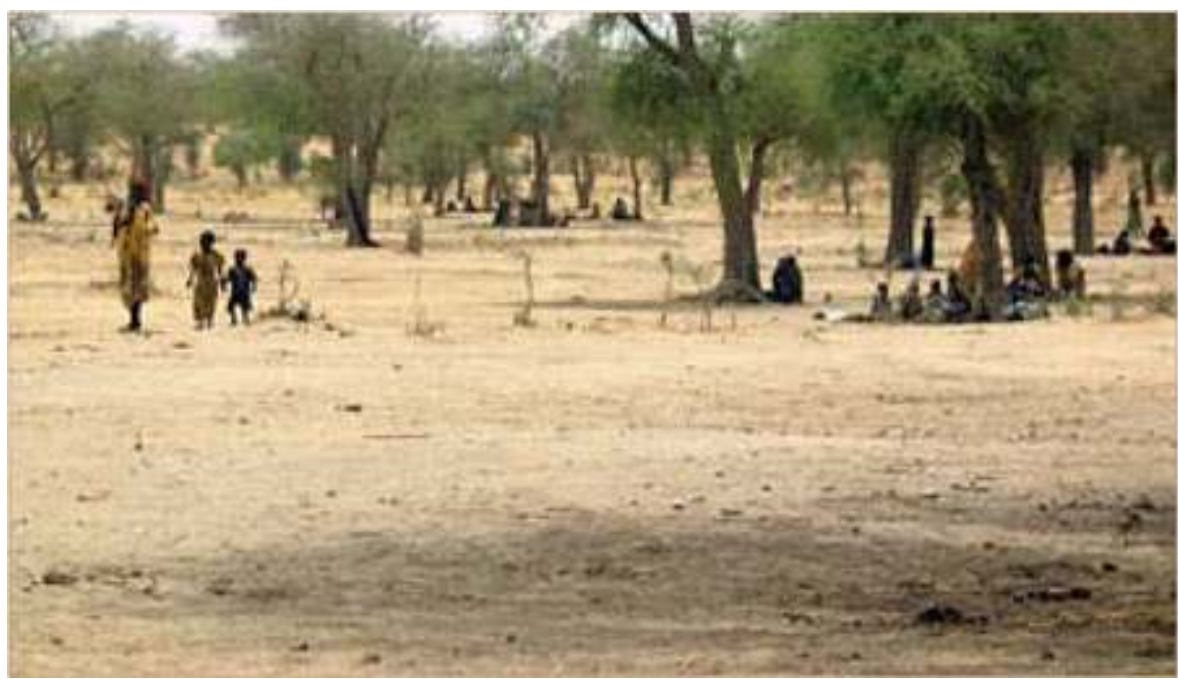

4. Le camp d'Am Nabak, au sud d'Iriba.

Ici, pas de distribution de tentes : le camp est « provisoire » depuis trois ans, en raison de l'absence d'eau sur place. Des camions-citernes approvisionnent quotidiennement ses 17000 réfugiés

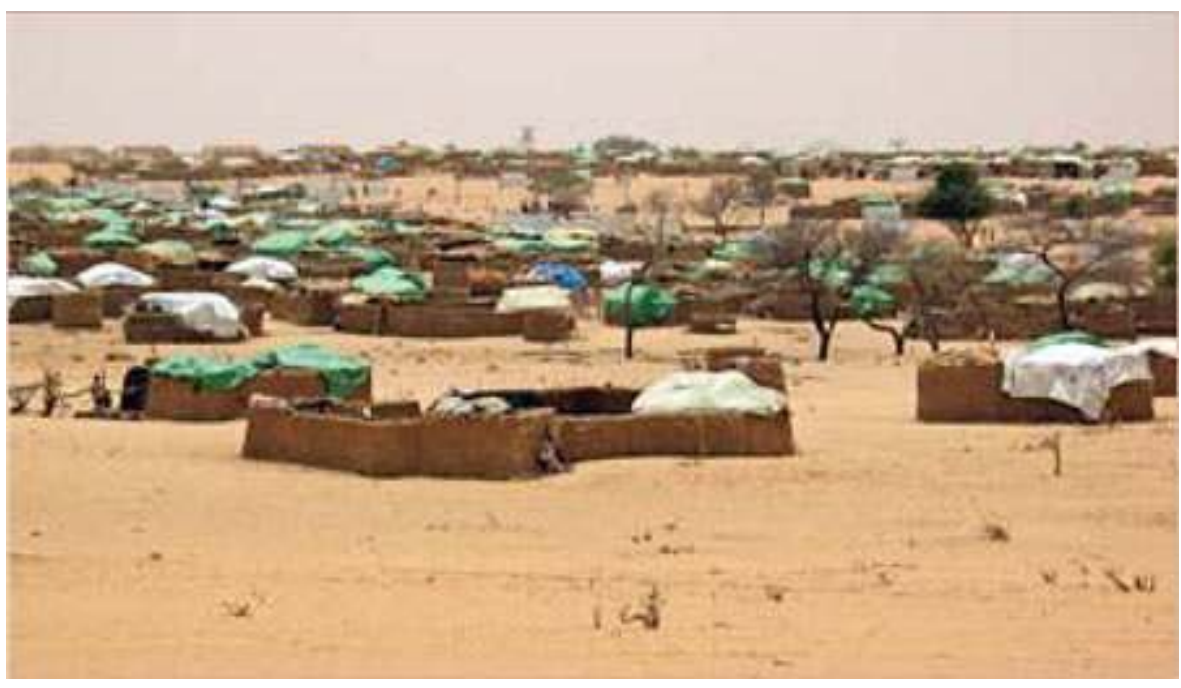

5. Des Tchadiens déplacés à Koloy, dans le lit du Ouadi Kadja. D’abord cantonnée dans la zone frontalière, l'insécurité s'étend à partir de 2006 à l'intérieur du Ouaddaï. 


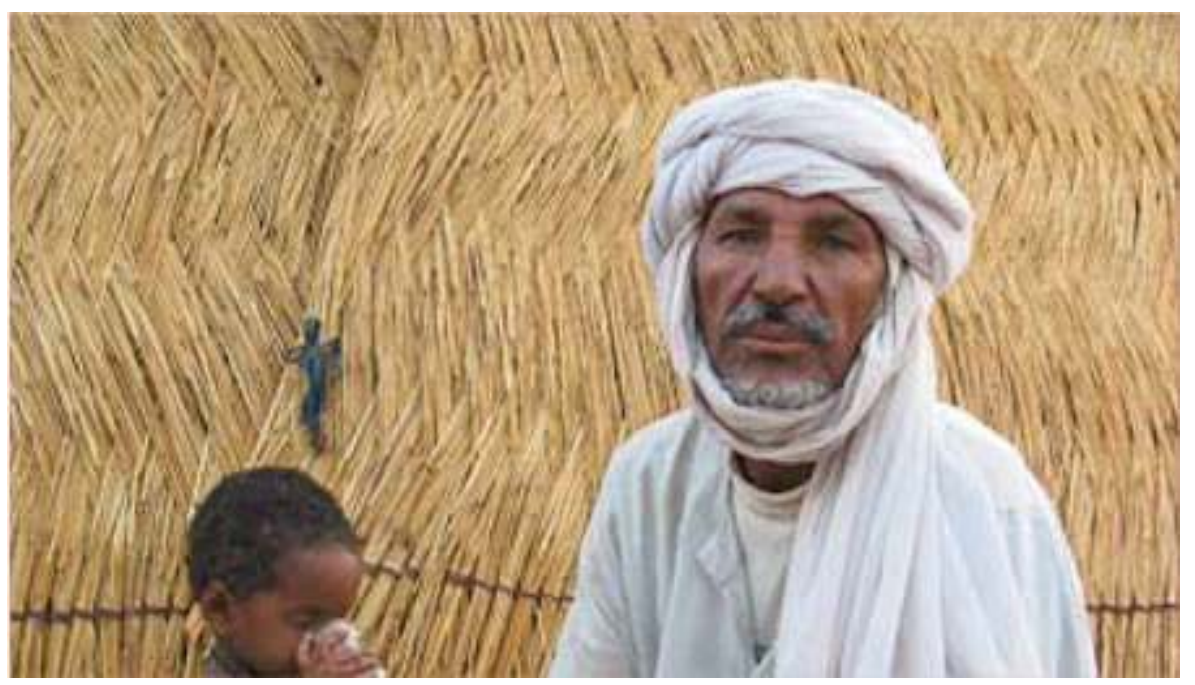

6. Arabes tchadiens sédentarisés autour de Goz Beïda.

Le camp de réfugiés de Djabal est installé sur les terres du village. A partir d'octobre 2006, l'entente entre les différentes communautés du département du Sila vole en éclat.

Dans l'est du Tchad, les déplacés tardent à être secourus. Début décembre 2006, l'insécurité provoque un recul de la présence humanitaire qui dure quelques mois. Puis des sites d'accueil sont peu à peu aménagés par les ONG présentes.

\section{L'EUFOR Tchad/RCA : une mission à haut risque}

La sécurité : c'est ce que réclament avant tout l'ensemble des populations présentes aujourd'hui dans l'est du Tchad. La saison des pluies a été exceptionnellement favorable aux cultures. Mais les violences contraignent à l'abandon des champs.

Et pourtant, l'arrivée de la future force européenne destinée à sécuriser la région n'est pas sans risque. Son effectif est faible pour les vastes étendues à couvrir. Des massifs rocailleux aux vallées ensablées des ouadis, le terrain est favorable à la guérilla. La saison des pluies terminée, les rebelles sont de retour: des combats contre l'armée gouvernementale reprennent à Goz Beïda, le 18 octobre 2007.

'est la composition de la force qui pose question. La France doit fournir la moitié du contingent. Or la France n'est pas seulement liée au gouvernement tchadien par un accord de coopération militaire; elle n'est pas seulement suspectée de partialité parce que le dispositif Epervier ${ }^{4}$ aide l'état-major tchadien dans sa lutte contre les rebelles : la France est militairement présente dans l'est tchadien depuis 1903, depuis qu'elle entreprit de "pacifier» l'empire du Ouaddaï. Elle vint à bout de l'empire par la force, décima l'élite intellectuelle musulmane et suscita parmi les peuples colonisés un repli culturel, une résistance aux valeurs qu'elle tentait de transmettre, par l'école notamment (Khayar, 1976 ; Mahamat Adoum Doutoum, 1983). Aujourd'hui, la présence occidentale au Ouaddaï, dans sa forme humanitaire ou militaire, est perçue à travers le prisme de cette confrontation, sans que les acteurs occidentaux en soient toujours conscients ${ }^{5}$.

Enfin, comment "protéger les civils» alors que les différents groupes rebelles recrutent dans les camps et les villages, alors que, partout, les communautés s'organisent en milices? Quand la violence se diffuse dans le corps social, les limites conceptuelles et temporelles de la guerre s'effacent. La « sécurisation » risque de n'être qu'apparente et éphémère, si les haines ne sont pas désarmées. 
Prévue pour une durée d'un an, l'EUFOR Tchad/RCA précède la mise en place au Darfour même d'une force « hybride » $\mathrm{ONU} /$ Union africaine de 26000 hommes. Une répétition, en quelque sorte, dont l'échec augurerait mal du règlement de la crise régionale.

\section{BIBLIOGRAPHIE}

Amnesty international, 2007. Tchad. « Sommes-nous citoyens de ce pays ? ». Les civils tchadiens laissés sans protection face aux attaques des Janjawids, 29 janvier, $13 \mathrm{p}$.

Bangoura M. T., 2005. Violence politique et conflits en Afrique : le cas du Tchad, L'Harmattan, Paris, $487 \mathrm{p}$.

Human Rights Watch, 2007. «They Came Here to Kill Us »: Militia Attacks and Ethnic Targeting of Civilians in Eastern Chad, janvier, $72 \mathrm{p}$.

International Crisis Group (ICG), 2006. « Tchad : vers le retour de la guerre ? ", Rapport Afrique ${ }^{\circ}$ 111, Nairobi/Bruxelles, 1er juin, 38 p.

Jean F., Rufin J.-C. (éd.), 1996. Economie des guerres civiles, Hachette, Paris, 595 p.

Khayar I. H., 1976. Le refus de l'école. Contribution à l'étude des problèmes de l'éducation chez les musulmans du Ouaddaï (Tchad), Librairie d'Amérique et d'Orient, Maisonneuve, Paris, 140 p.

Mahamat Adoum Doutoum, 1983. La colonisation française et la question musulmane au Tchad. Exemple du sultanat du Ouaddaï (1895-1946), thèse de 3e cycle, Université de Paris-Sorbonne, 310 p.

Marchal R., 2006. «Tchad/Darfour : vers un système de conflits », Politique Africaine $n^{\circ} 102$, juin, p. 134-154.

\section{NOTES}

1.. 233100 selon le HCR le 30 septembre 2007 .

2.Action contre la faim, 8 novembre 2006.

3.En mai 2007, le HCR évalue à 45000 le nombre de Tchadiens réfugiés au Darfour ; il s'agit essentiellement d'Arabes nomades.

4.Mis en place en 1986 pour contrer l'expansionnisme libyen au Tchad, le dispositif Epervier (environ 1200 hommes présents à N’Djamena, Abéché et Faya-Largeau) vise désormais à protéger les quelques 3000 ressortissants français et étrangers présents sur place et à soutenir l'armée nationale tchadienne. Il succède à plusieurs interventions françaises dans les guerres du Tchad depuis 1965.

5.La virulence des réactions locales aux agissements de «L'Arche de Zoé » est à interpréter dans ce contexte. Des membres de cette association ont été arrêtés le 25 octobre 2007 à Abéché alors qu'ils s'apprêtaient à transporter illégalement vers la France 103 enfants originaires de villages proches de la frontière soudanaise. 


\section{RÉSUMÉS}

Une mission conjointe ONU/UE est attendue pour novembre 2007 dans l'est du Tchad, où elle doit sécuriser les camps qui hébergent 230000 réfugiés du Darfour et 180000 déplacés tchadiens. Mais la persistance des rébellions dans la région témoigne de l'absence de règlement de la crise politique au Tchad. En outre, l'objectif de sécurisation risque de se heurter à la dissémination de la violence dans le corps social.

A joint UNO/EU mission is expected in Eastern Chad in November 2007, to guard the camps which hold 230000 refugees from Darfur and 180000 displaced Chadians. But the continued rebellions in the region highlight the absence of a settlement to the political crisis in Chad. Moreover, the attempt to guard may run up against the violence spreading through society.

\section{INDEX}

Mots-clés : réfugiés, Tchad oriental, déplacés, conflit, EUFOR Tchad/RCA

Keywords : refugees, Eastern Chad, displaced people, conflicts, EUFOR Chad/CAR

\section{AUTEUR}

\section{JOHANNE FAVRE}

Johanne Favre (johannefavre@yahoo.fr) est agrégée de Géographie et doctorante à l'Université de Paris I, rattachée à l'UMR PRODIG-CNRS.

Elle a récemment publié :

" Abéché, porte de l'Orient ». Atlas du Tchad, Les éditions J.A., 2006, p. 54-55 et.

« Marginalité de l'Etat et violences sociales au Far Est (Tchad oriental) ». Bulletin de l'Association de Géographes Français, Géographies, Septembre 2007, p.357-365. 